\title{
El estrés crónico y la Terapia Cognitiva Centrada en Mindfulness: Una nueva dimensión en psiconeuroinmunología
}

Manolete S. Moscoso

Universidad de Florida del Sur

Recibido: 30 de junio del 2010 / Aprobado: 12 de agosto del 2010

En el presente artículo se hace una revisión del concepto de estrés crónico desde una perspectiva psiconeuroinmunólogica. Se examina un extenso número de estudios multidisciplinarios que demuestran las interacciones entre el cerebro y los sistemas nervioso, endocrino e inmune en el desarrollo de enfermedades. Se presenta una nueva visión acerca del estrés y se hacen las distinciones correspondientes en relación con la respuesta fisiológica, emocional, comportamental y estilo de vida del individuo. Se examina el impacto negativo del estrés crónico con un énfasis especial en la enfermedad del cáncer, y finalmente se hace referencia al programa de la Terapia Cognitiva Centrada en Mindfulness (TCCM) como una forma de intervención biopsicosocial promisoria para contrarrestar los efectos nocivos del estrés crónico.

estrés crónico / calidad de vida / cáncer / mindfulness / psiconeuroinmunología

\section{Chronic stress and the Mindfulness-Based Cognitive Therapy: A new dimension in psychoneuroimmunology}

This article reviews the concept of chronic stress from the psychoneuroimmunologic point of view. We examined an extensive multidisciplinary studies and research reporting conclusive evidence of the interactions between the brain, the nervous, endocrine and immune systems in the development of disease. We discussed the negative impact of chronic stress on health and disease. A new vision about stress is presented, taking into account the physiological, emotional and behavioral responses to stress, as well as the life style of the individual. We pointed out the negative impact of chronic stress with an emphasis on cancer, and finally we made a reference to the role of Mindfulness Based Cognitive Therapy (MBCT) as a promissory biopsychosocial intervention to buffer chronic stress.

chronic stress / quality of life / cancer / mindfulness / psychoneuroimmunology. 
El gran avance científico producido por las investigaciones en neuroendocrinología y psiconeuroinmunología en lo que va del siglo XXI, nos permite entender con mayor amplitud la respuesta fisiológica del estrés y sus interacciones multidireccionales con el cerebro, los sistemas nervioso, endocrino e inmune, y sus consecuencias en la salud del individuo (Moscoso, 2009; Steinman, 2004). A pesar de este significativo progreso, el concepto de estrés continúa siendo sumamente ambiguo. Presenta muchas connotaciones y poca utilidad para entender cómo el organismo se adapta a las demandas de la vida diaria y a situaciones devastadoras que ocurren de manera ocasional. Mientras para algunos individuos el concepto de estrés refleja un estado de preocupación, temor, irritabilidad, tristeza y dificultad para manejar adecuadamente las situaciones que causan frustración, para otros el estrés es un reto que motiva para la obtención de logros y metas en la vida. En el primer caso, hacemos referencia a un término popular denominado "estresado", el cual sugiere la naturaleza crónica de un estado negativo reconocido clínicamente como distrés emocional (Moscoso, McCreary, Goldenfarb, Knapp \& Rohr, 1999; Moscoso \& Reheiser, 2010).

Una distinción muy importante en la noción de estrés es el proceso de evaluación cognitiva descrita por Lazarus y Folkman (1984), que toma en consideración un elemento importante como es la percepción por parte del individuo. El estrés psicológico es originado por estresores externos o internos y está sujeto a una evaluación cognitiva denominada appraisal. Dichos autores la definen como la interacción entre una amenaza externa, la evaluación cognitiva de amenaza (appraisal primario) y los recursos personales percibidos para enfrentar tal amenaza (appraisal secundario).

Otra distinción fundamental en el estudio del estrés demuestra que los estresores y las respuestas del estrés son conceptos totalmente distintos. Es importante reconocer la respuesta emocional del estrés, la cual está caracterizada por síntomas de ansiedad, irritación, ira, preocupación, tristeza, pánico y estados de desesperanza. El ingrediente principal de la respuesta emocional del estrés es de naturaleza cognitiva debido al hecho de que el "estímulo externo deberá ser percibido como estresante". El modelo descrito por Lazarus y Folkman propone que en respuesta a un estímulo externo de naturaleza estresante, el individuo evalúa cognitivamente la posible amenaza que este evento representa y los recursos propios o la capacidad para responder a dicho estímulo. Este proceso de evaluación cognitiva primaria y secundaria determina la forma e intensidad de la reacción emotiva en relación con el estímulo externo. Es importante indicar que la evaluación cognitiva por parte de un individuo varía, dependiendo de los rasgos de personalidad, autoefica- 
cia percibida (Bandura, 1997), experiencia previa con el estímulo estresante, y nivel de soporte social. Por lo tanto, la respuesta emocional del individuo no puede ser generalizada, y varía aun cuando las condiciones de estímulos estresantes pudieran ser semejantes (Lazarus, 1993).

Científicos interesados en la respuesta emocional del estrés fijan su atención en eventos estresantes de carácter universal. Al plantear el diseño de sus investigaciones, seleccionan situaciones de estrés que generalmente afectan psicológicamente a la gran mayoría de personas. Entre este tipo de eventos encontramos las situaciones de divorcio, encarcelamiento, exámenes académicos, pérdida de los seres queridos, cuidado permanente de los familiares con discapacidad física, diagnóstico de enfermedades crónicas (Martin \& Dean, 1993; Moscoso, 1995; Spielberger \& Moscoso, 1996), dificultades financieras y otras que típicamente causan niveles elevados de distrés emocional. Es importante mencionar que la respuesta emocional del estrés puede también variar debido a los diferentes estilos de afrontamiento. Finalmente, merece destacarse que la respuesta emocional del estrés es básicamente de naturaleza transitoria y temporal, en todo caso nos estamos refiriendo a un proceso de estrés agudo.

Una tercera distinción en el análisis de este importante campo de estudio se refiere a la respuesta fisiológica del estrés, conocida también como fightor-flight. La respuesta fisiológica del estrés fue descrita inicialmente por Walter Cannon en 1915, al referirse a la respuesta del animal dentro del laboratorio de experimentación ante una situación de amenaza o presión externa. Cannon, en su obra Cambios corporales en situaciones de dolor, hambre, temor y rabia, explica en forma detallada que la respuesta fisiológica del estrés nos permite reaccionar ante una situación de emergencia con todo nuestro potencial físico, superar el peligro y lograr un proceso de adaptación frente a las circunstancias que nos rodean. Este principio de adaptación homeostática fue el concepto central en el modelo de estrés descrito por Cannon en 1932 con la publicación de su obra La sabiduría del cuerpo, refiriéndose a la estabilidad de los sistemas fisiológicos que mantienen la vida en estado de armonía u homeostasis.

El concepto de homeostasis como proceso de adaptación, inicialmente presentado por Walter Cannon, fue ampliamente descrito por Hans Selye en 1936 denominándolo Síndrome de adaptación general. Selye caracterizó el síndrome de adaptación general como una respuesta fisiológica representada por tres fases definidas. La primera fase denominada Reacción de Alarma, en la cual las glándulas adrenales producen adrenalina y cortisol con el propósito de restaurar la homeostasis. Dicha restauración de la home- 
ostasis inicia la segunda fase llamada Resistencia, en la cual la adaptación del organismo llega a un estado óptimo. Finalmente, si el estresor persiste, se inicia una tercera fase conocida como Agotamiento, en la que el organismo abandona el proceso de adaptación y culmina en la enfermedad o la muerte.

El propósito de este artículo es presentar distinciones importantes del concepto de estrés y explicar las diferencias fundamentales en relación con el concepto de estrés crónico y sus interacciones con el cerebro, los sistemas nervioso, endocrino e inmune; y sus efectos sobre la salud del individuo. Asimismo, pone énfasis en la utilización de la Terapia Cognitiva Centrada en Mindfulness como un programa de intervención biopsicosocial en la reducción del estrés crónico y su utilización en el campo de la psiconeuroinmunología.

\section{EFECTOS DEL ESTRÉS SOBRE El SISTEMA INMUNE}

Es muy importante tener en cuenta que los estudios de la respuesta fisiológica del estrés realizados por Cannon y Selye fueron llevados a cabo estrictamente con animales en el laboratorio de experimentación, mientras que los estudios actuales en psiconeuroinmunología incluyen además muestras con seres humanos. Sin lugar a dudas, los estudios de Lazarus (1993) y la inclu- sión del concepto de evaluación cognitiva (appraisal) y estrés percibido han permitido desarrollar nuevas líneas de investigación científica, indicándonos que no todas las situaciones de estrés evocan el mismo tipo de respuestas fisiológicas, como se menciona en los estudios de Cannon y Selye.

También es importante indicar que los estudios científicos del estrés en seres humanos demuestran la necesidad de tomar en consideración los factores ambientales, culturales, actitudinales y rasgos de personalidad que cumplen un rol mediador y modulador en la respuesta fisiológica del estrés (Goldstein \& Eisenhofer, 2000). Asimismo, está empíricamente demostrado que la respuesta del estrés puede ser diferente cuando se tiene en cuenta el sexo del individuo. En este sentido, existen reportes que indican que el sexo masculino reacciona de manera diferente cuando se trata de manejar las distintas situaciones de amenaza o estresores (Taylor et al., 2000).

En términos generales, la respuesta fisiológica del estrés es activada por un estresor. Esta respuesta fisiológica cumple una misión esencialmente protectora, pro homeostática, cuya intención es mantener un balance homeostático en el organismo, y es activada en forma instantánea. La respuesta fisiológica del estrés se inicia en el hipotálamo con la producción de la hormona liberadora de corticotropina (CRH) dirigida al sistema circulatorio de la 
glándula pituitaria, la cual segrega la hormona adenocorticotropa (ACTH) a través del eje hipotálamo-pituitariasuprarrenal (eje HPA) produciendo hormonas glucocorticoides, especialmente cortisol (McEwen, 2007).

De igual manera, la respuesta fisiológica del estrés activa el eje simpático-suprarrenal medular (SAM), el cual genera la secreción de catecolaminas como la adrenalina y noradrenalina. Estas hormonas generan un aumento en la concentración de glucosa en la sangre facilitando un mayor nivel de energía, oxígeno, alerta, fuerza muscular y resistencia al dolor; todo este proceso se manifiesta en cuestión de minutos. La liberación de dichas hormonas causadas por un estresor permite el inicio de un proceso de interacción inmediata con otras áreas del cerebro y el sistema nervioso autónomo, a través de los sistemas simpático y parasimpático, enlazando la experiencia del estrés con los componentes psicofisiológicos y bioquímicos de la emoción y preparando el organismo para un estado de alerta (Padgett \& Glaser, 2003).

Figura 1

Efectos del estrés crónico sobre los sistemas nervioso, endocrino e inmune

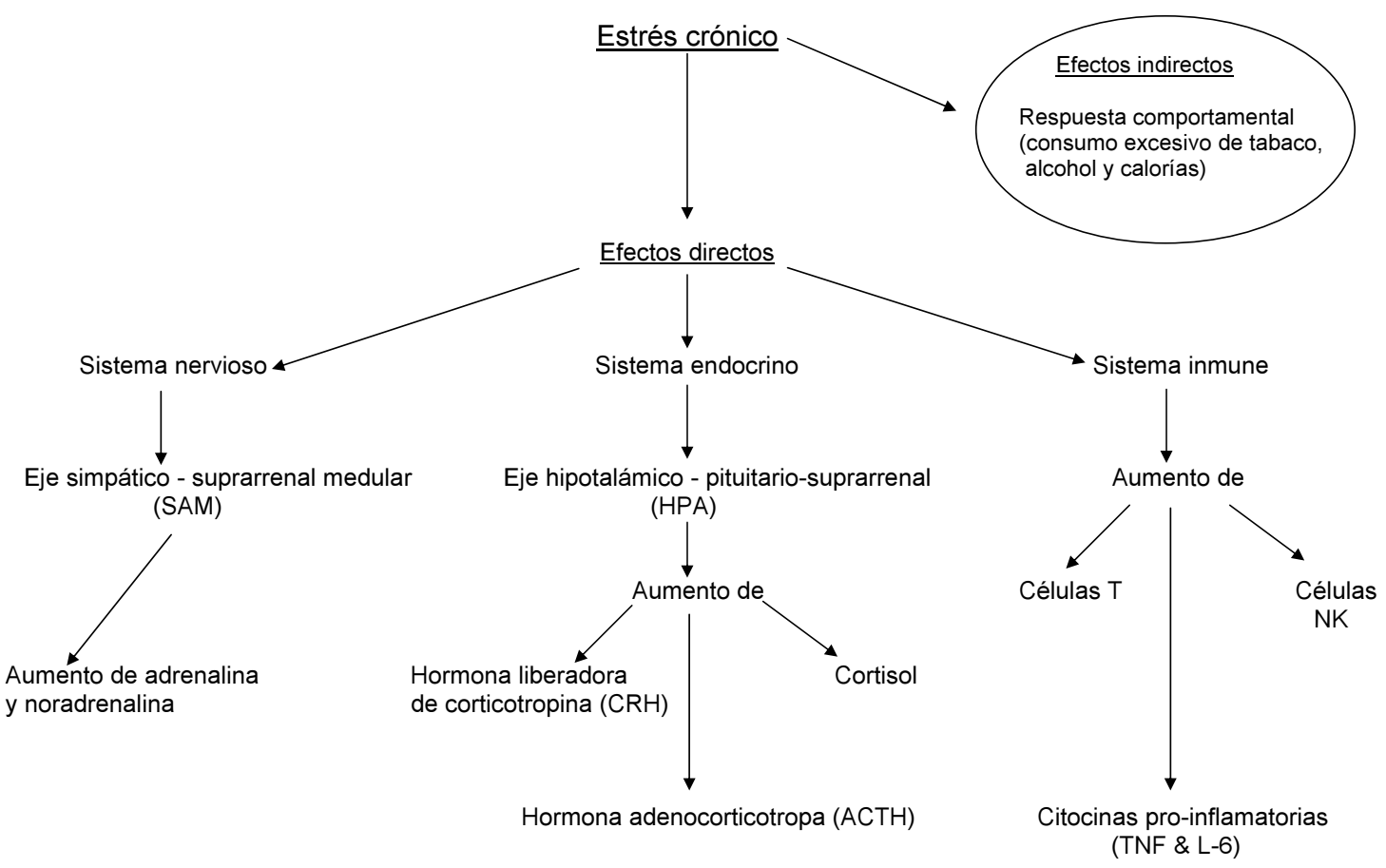


Sobre la base del reporte de un gran número de estudios publicados en el campo de la psiconeuroinmunología, está científicamente establecido que el estrés libera otros factores y hormonas neuroendocrinas que regulan los sistemas nervioso e inmune. Estas incluyen la hormona del crecimiento (GH), la prolactina, la vasopresina, el glucagón, las endorfinas, las encefalinas y la oxitocina, entre otras. El cortisol liberado por las glándulas suprarrenales facilita la preparación del sistema inmune para manejar sus defensas contra bacterias, virus, heridas e inflamaciones. Esta breve descripción de las diferentes interacciones cerebrales con el resto de los sistemas nervioso, neuroendocrino e inmune nos permite comprender que los mecanismos psicofisiológicos de la respuesta del estrés juegan un rol esencial en el proceso de adaptación y supervivencia del individuo sobre la base de los diferentes mecanismos de autorregulación homeostática. Asimismo, es vital entender que el incremento de cortisol, adrenalina, noradrenalina y las hormonas generadas durante la situación de estrés agudo cumplen una función eminentemente protectora y de supervivencia, siendo la función central y primordial mantener el equilibrio homeostático, y como consecuencia la salud del individuo. Estos sistemas reguladores de glucocorticoides y hormonas liberadas durante la respuesta fisiológica del estrés facilitan el proceso de adaptación del organismo a los diferentes eventos estresantes, a los cuales estamos expuestos diariamente (McEwen \& Wingfield, 2003).

\section{ESTRÉS CRÓNICO Y ESTILO DE VIDA: UN NUEVO PARADIGMA EN INVESTIGACIÓN}

Dentro de los modelos tradicionales del estudio del estrés, es común encontrar literatura científica y una amplia descripción de la respuesta fisiológica y la respuesta emocional del estrés. Un nuevo paradigma en el estudio del estrés percibido incluye la respuesta comportamental del estrés, la cual toma en consideración el estilo de vida del individuo y el medio ambiente en el cual este se desenvuelve. El estilo de vida del individuo y el medio ambiente en el que interactúa son factores centrales, mediadores y moduladores en el proceso de cronicidad del estrés. Frente a la experiencia diaria de estresores, nuestro comportamiento puede permitirnos escoger un estilo de vida saludable que favorezca la reducción del estrés y lograr un buen nivel de calidad de vida. De otra manera, también existe la posibilidad de asumir un estilo de vida que ayude a acentuar y experimentar un mayor nivel de distrés emocional, contribuyendo al desarrollo de un patrón conductual caracterizado por reacciones emocionales y síntomas de estrés de carácter crónico, como fumar y tomar licor en exceso, el elevado consumo de calorías y grasas saturadas, la falta de entrenamiento físico, las largas horas de trabajo, el aislamiento 
social y una gran falta de actividades relacionadas con la relajación y el descanso corporal (Epel, Lapidus \& McEwen, 2000).

Es necesario reiterar que el cerebro no solamente tiene un enorme control sobre el sistema nervioso autónomo, sistema endocrino y sistema inmune en la regulación de neurotransmisores, glucocorticoides, hormonas y citocinas, sino también sobre el comportamiento del individuo y su forma de relacionarse con el medio ambiente. En este sentido, el biólogo genético Craig Venter, Ph.D., durante su presentación Los genes tienen un impacto mínimo en los resultados de vida, ofrecida en la Conferencia de Liderazgo para el Siglo XXI, que se realizó en marzo del 2008, indicó lo siguiente:

La biología humana es mucho más complicada de lo que nos imaginamos. Todos hablan de los genes que reciben de su madre y de su padre, para explicar un rasgo $u$ otro. Pero en realidad, esos genes tienen un impacto mínimo en los resultados de vida. Nuestra biología es demasiado complicada para ello y alterna con cientos de miles de factores independientes. Los genes no son absolutamente nuestra suerte o destino. Ellos nos pueden dar una información útil acerca del riesgo para contraer una enfermedad, pero en la mayoría de casos estos genes no van a determinar el actual curso de la enfermedad, o la incidencia de que alguien la adquiera. Mucho de nuestra biología proviene de las complejas interacciones de todas las células y proteinas interactuando conjuntamente con factores ambientales, no guiadas directamente del código genético (Venter, 2008).
Las palabras textuales de Venter describen claramente la importancia de los factores ambientales y estilos de vida en el proceso de mantenimiento de la salud y adquisición de enfermedades.

\section{EL ESTRÉS CRÓNICO COMO CAUSA DE ENFERMEDADES}

Estudios recientes en psiconeuroinmunología y neuroendocrinología nos demuestran la necesidad de reevaluar los modelos conceptuales tradicionales acerca del estrés. En primer lugar, es necesario dejar establecido que el modelo cognitivo del estrés percibido tiene un correlato eminentemente fisiológico en el lóbulo frontal del cerebro, por lo que es necesario reconocer que la experiencia del estrés se inicia en el cerebro, afecta a este y a su vez al resto de los sistemas que conforman nuestro organismo (McEwen, 1998; Sapolsky, 1996). En este sentido, podemos afirmar de manera categórica que el cerebro es el órgano central de la respuesta fisiológica, emocional y comportamental del estrés. La percepción del individuo como ingrediente cognitivo elaborado en el lóbulo frontal del cerebro determina ciertamente lo que es "estresante".

En segundo lugar, estudios realizados en neuroendocrinología demuestran que cuando la respuesta del estrés percibido se transforma en un proceso crónico, esta respuesta facilita el inicio de un estado de distrés emocional en el 
cual se observa una excesiva utilización o, a veces, un manejo ineficiente de hormonas, corticoesteroides y catecolaminas por parte de los ejes neuroendocrinos, es decir el eje hipotalámico-pituitario-suprarrenal (HPA Axis) y el eje simpático-suprarrenal-medular (SAM Axis) (McEwen, 2007). Este estado de distrés emocional causado por el estrés crónico produce un impacto negativo en el sistema nervioso activando cambios bioquímicos y un desbalance hormonal que repercute en los sistemas endocrino e inmune (Dhabhar $\&$ McEwen, 1997). Ciertamente, este proceso nos permite entender y apreciar la naturaleza multidireccional de las interacciones entre los estresores, el cerebro y los sistemas endocrino e inmune (Sapolski, Romero \& Munck, 2000).

El avance científico en el campo de la psiconeuroinmunoendocrinología, particularmente en las dos últimas décadas, ha facilitado un mejor entendimiento acerca de la importancia del ambiente social y estilos de vida como factores mediadores del impacto negativo del estrés crónico en la salud física y mental del individuo. En la actualidad está científicamente establecido que este impacto negativo propicia el desarrollo de un significativo número de enfermedades de carácter sistémico, como inflamación crónica, artritis reumatoide, fibromialgia, fatiga de las glándulas suprarrenales, enfermedades cardiovasculares, hipertensión, diabe- tes tipo II, obesidad, síndrome metabólico, asma, cáncer, depresión y otras enfermedades inmunosupresoras. Por lo que podemos observar hasta la fecha, el deterioro de la salud es el resultado de un proceso permanente de interacción multidireccional a través del lóbulo frontal del cerebro (estrés percibido), del sistema nervioso autónomo, del sistema endocrino y del sistema inmune (Taub, 2008).

Son justamente estos cuatro sistemas los que están preparados para proteger el organismo con el propósito de lograr un equilibrio homeostático ante una situación aguda de estrés; sin embargo, irónicamente, son estos mismos cuatro sistemas los que contribuyen a crear un desbalance bioquímico durante la experiencia de estrés crónico. Es importante dejar establecido que el desarrollo de un estado de estrés crónico facilita, a la vez, estilos de vida poco saludables que únicamente contribuyen a empeorar la situación de estrés y generar niveles más agudos de distrés emocional. Nos referimos como ejemplo a estilos de vida que fomentan el consumo excesivo de alcohol, tabaco y drogas, y una ingesta elevada de calorías y grasas saturadas (Armeli,Todd \& Mohr, 2005). Este nuevo punto de vista acerca del concepto de estrés crónico nos impone la necesidad de dar una mayor importancia al ambiente social y a los estilos de vida saludables adquiridos por el individuo, como también nos exige otorgar priori- 
Figura 2

Una nueva visión del estrés

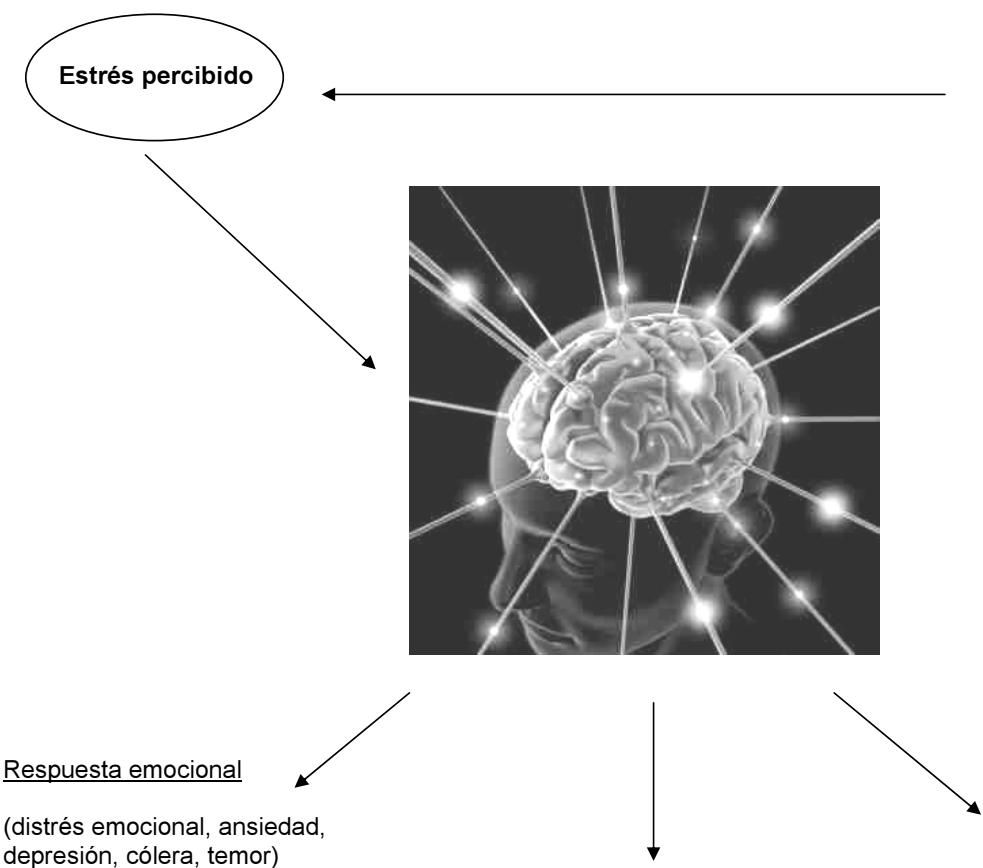

Diferencias individuales

(personalidad, cultura, educación, género, estrato socio-económico)

Repuesta fisiológica

( HPA eje, SAM eje)

dad al estudio de la cronicidad en la experiencia del estrés percibido representada por niveles significativamente elevados de distrés emocional.

Lo indicado en líneas anteriores nos permite plantear tres interrogantes para ser evaluadas empírica y científicamente en el campo de la psiconeuroinmunología:

- ¿En qué momento, y cómo, los efectos neuroendocrinos e inmunológicos provocados por el estrés dejan de favorecer la adaptación del individuo por ser esencialmente protectores del proceso homeostático?

- ¿Cómo es posible que el mismo mecanismo destinado a protegernos y mantener el principio de homeostasis tome de pronto un curso totalmente opuesto e inicie un proceso de deterioro de la salud del individuo?

- ¿Qué podemos hacer, en términos de intervenciones psicológicas, para controlar los efectos nocivos del estrés crónico en la salud y prevenir enfermedades? 
Para responder estas preguntas es necesario tomar en consideración el impacto negativo del estrés percibido en términos de niveles de distrés emocional y sus efectos nocivos en el comportamiento del individuo. Asimismo, se requiere prestar atención al periodo prolongado de tiempo experimentando situaciones de estrés que determinan el inicio de un proceso que típicamente lo conceptualizamos como estrés crónico o distrés emocional (Moscoso, McCreary, Goldenfarb, Knapp \& Reheiser, 2000; Moscoso \& Reheiser, 2010). Hace relativamente poco tiempo, científicos del campo de la neuroendocrinología presentaron una visión más amplia de la respuesta del estrés que incluye el concepto de alostasis, refiriéndose a "estabilidad a través del cambio" (Sterling \& Eyer, 1988). Este nuevo modelo conceptualiza la existencia de los sistemas nervioso, endocrino e inmune en un estado de fluidez, los cuales responden a las demandas ambientales. El exceso de demandas ambientales genera perturbaciones en la habilidad y capacidad del organismo para responder a estas demandas y retornar de manera natural a un estado de homeostasis. Este desbalance generado por el exceso crónico de demandas externas e internas en la vida diaria de un individuo produce un desgaste en las funciones bioquímicas de los sistemas antes mencionados ocasionando un estado de estrés crónico conceptualizado como allostatic load (McEwen, 2006).
Las diferencias conceptuales entre las respuestas fisiológica y emocional del estrés y el estrés crónico facilita una reinterpretación del síndrome de adaptación general de Selye y sugiere la concepción de un nuevo modelo conceptual del estrés en términos de alostasis y allostasis load. Este nuevo paradigma conceptual del estrés nos permite afirmar que son los mediadores bioquímicos del estrés percibido (catecolaminas, glucocorticoides y citocinas) los que generan un cambio de 180 grados en cuanto a su función protectora debido a los efectos acumulativos "estresantes" en la vida diaria de un individuo, causando un desbalance en el cerebro, particularmente la amígdala y el hipocampo, y los sistemas nervioso, endocrino e inmune, los cuales alteran el equilibrio homeostático y causan un estado de estrés crónico o allostasis load. Los efectos acumulativos del estrés crónico dan como resultado la alteración de los mediadores primarios de la respuesta fisiológica del estrés, por mencionar los principales: cortisol, adrenalina, insulina, vasopresina, endorfinas y citocinas, reflejando un aumento en la producción de algunas de ellas, o una insuficiencia en la liberación de otras, determinando en última instancia la pérdida de la salud (Koob, 1999).

Esta alteración en los sistemas reguladores (HPA axis y SAM axis) productores de glucocorticoides y hormonas, producen un desgaste en las múlti- 
ples funciones cerebrales, endocrinas e inmunológicas, predisponiendo el desarrollo de las alteraciones físicas y mentales antes mencionadas. En este sentido, los resultados de un significativo número de estudios empíricos dejan científicamente establecido que el estrés crónico produce alteraciones en las neuronas del hipocampo, generando problemas de la memoria (McEwen, 2001). Asimismo, se observa una supresión en las defensas del sistema inmune y toda una constelación de síntomas psicofisiológicos como la fatiga de las glándulas suprarrenales causada específicamente por la reducción de cortisol. Está establecido que el estrés crónico o el exceso de distrés emocional tienen una influencia directa en los procesos inflamatorios del organismo debido a la elevación crónica de citocinas pro-inflamatorias, los cuales, a su vez, son causantes directos de alergias respiratorias, particularmente asma, artritis reumatoide y enfermedades cardiovasculares; como también depresión, insomnio y fatiga crónica causada por una reducción en los niveles de cortisol (MacPherson, Dinkel \& Sapolski, 2005). Desde el punto de vista metabólico, el estrés crónico induce al consumo excesivo de calorías, que produce el incremento de cortisol, glucosa e insulina, causantes de la obesidad, la diabetes tipo II y un significativo número de enfermedades cardiovasculares (Dallman, 2003; Epel, Lapidu \& McEwen, 2000).
UN NUEVO MODELO TERAPÉUTICO PARA EL MANEJO DEL ESTRÉS CRÓNICO: EL MINDFULNESS

La tercera interrogante planteada en relación con el estrés crónico debe ser respondida sobre la base de un modelo de intervención psicológica con un significativo énfasis en prevención y terapias integrales que promuevan el bienestar y reduzcan los niveles de distrés emocional. De igual manera, este nuevo paradigma necesita tomar en consideración todos los factores del modelo biopsicosocial que cumplen un rol importante en el proceso de desarrollo del estrés crónico y su impacto en el cerebro, los sistemas nervioso, endocrino e inmune, los factores ambientales y el estilo de vida del individuo. Un adecuado proceso de afrontamiento $\mathrm{y}$ manejo del estrés crónico incluye la participación en actividades que promueven un estilo de vida saludable y de mejora de la calidad de vida del individuo. Por lo tanto, la incorporación de un plan nutricional balanceado, programas moderados de ejercicios físicos y técnicas que facilitan la respuesta de relajación son vitales y de gran necesidad.

La práctica de tomar conciencia y prestar atención (mindfulness) a los síntomas de distrés emocional que experimenta un individuo bajo estrés crónico, facilita la labor terapéutica y posibilita las modificaciones necesarias para mejorar su estilo de vida. En 
este sentido, el programa de la Terapia Cognitiva Centrada en Mindfulness (TCCM ) ha ganado un gran nivel de aceptación en el campo de la medicina, la psicología y la psiconeuroinmunología. El propósito de este programa de intervención terapéutica es entrenar al individuo en la respuesta de relajación, reducir los niveles de estrés crónico y modificar los patrones de conducta autodestructivos. Asimismo, permite desarrollar un estilo de vida saludable con la intención de promover la calidad de vida del paciente (Moscoso, Reheiser \& Hann, 2004).

El manejo del estrés crónico a través de la práctica del mindfulness facilita el aprendizaje de la respuesta de relajación, así como un entendimiento más profundo de los síntomas emocionales, físicos y comportamentales que caracterizan los estados de estrés crónico. Es importante destacar que la TCCM es actualmente uno de los enfoques cognitivos de mayor desarrollo y aceptación en Estados Unidos y Europa, no solamente para el manejo del distrés emocional causado por el diagnóstico de enfermedades crónicas y terminales, sino también para obtener un beneficio inmunológico y neuroendocrino que promueven el restablecimiento de la salud y contribuyen a un mejor control de los síntomas causados por los tratamientos médicos (Lengacher et al., 2009). Por otro lado, la TCCM permite asistir a pacientes que experimentan niveles significativamente elevados de depresión y contrarrestar la posible recurrencia de esta enfermedad.

El mindfulness puede ser descrito como una "forma de percibir" y expandir nuestro nivel de conciencia; una manera particular de "prestar atención", momento a momento, a las experiencias que nos toca vivir en la vida diaria, de tal manera que logremos desarrollar un nivel de intimidad con nuestros propios sentidos, nuestro cuerpo y nuestras experiencias personales. Esta forma particular de tomar conciencia nos facilita un mayor y más profundo entendimiento de nuestras propias vivencias y dificultades cotidianas. El mindfulness nos permite centrar la atención y la conciencia en nuestro cuerpo a través de la respiración; en nuestra mente a través del pensamiento; y en nuestro entorno a través de nuestros sentidos.

La práctica del mindfulness nos posibilita experimentar el momento presente. Importa el aquí y ahora, esencialmente uno de los fundamentos de la terapia cognitiva. El mindfulness como forma de meditación tiene sus raíces en una de las más antiguas prácticas de meditación budista denominada vipasana. En este sentido, la meditación y la respuesta de relajación tienen una tradición clínica muy amplia en el campo de la psicología de la salud, particularmente en biofeedback y en la respuesta de relajación de Benson. Uno de los postulados fundamentales del modelo terapéutico basado en mindfulness es cuestionar el constante esfuerzo por 
"eliminar" el estrés y la reacción emocional a este fenómeno. Por el contrario, bajo este modelo terapéutico aprendemos que el estrés y las experiencias negativas que se presentan en nuestras vidas tienen una razón de ser, los cuales únicamente incrementan nuestro valor adaptativo como seres humanos. La creencia obstinada de "vivir huyendo" del estrés y del dolor emocional que las experiencias diarias de la vida producen solo contribuye a elevar los niveles de tensión, ansiedad, temor, preocupación, ira y hostilidad. En la medida en que modificamos nuestra actitud hacia las dificultades que nos ofrece la vida y aprendemos a ser conscientes de que el dolor y el placer son experiencias genuinamente humanas, tendremos la posibilidad de lograr un significativo nivel de aceptación y congruencia en nosotros mismos.

De manera particular, los beneficios terapéuticos del programa TCCM en pacientes con cáncer vienen siendo demostrados científicamente sobre la base de estudios llevados a cabo durante la última década. Bishop (2002) reportó que dicho programa fue eficaz en lograr que los pacientes entrenados puedan manejar adecuadamente sus niveles de distrés emocional y reacciones somáticas frente a situaciones de estresores. Speca y colaboradores (2000) demostraron que el mindfulness es efectivo en la reducción de los síntomas del distrés emocional, en comparación con el grupo de control. Los estu- dios dirigidos por Linda Carlson y colaboradores (2001, 2003, 2004, 2007) demuestran que el programa del mindfulness mejora la calidad de vida de pacientes con cáncer y reduce los niveles de distrés emocional. Asimismo, los estudios de Carlson reportaron una regulación óptima de los patrones de cortisol en los pacientes asignados al grupo de tratamiento, en comparación con los pacientes asignados a los grupos de control. Un estudio dirigido por Moscoso, Reheiser y Hann (2004) demostró una reducción significativa en los niveles de ansiedad, depresión y distrés emocional en un grupo de pacientes con cáncer de mama.

La experiencia de distrés emocional en pacientes con cáncer activa los sistemas regulatorios de la respuesta de estrés, eleva las secreciones hormonales, y altera las funciones inmunológicas del paciente (Kiecolt-Glaser, McGuire \& Robles, 2002). Como indicáramos en líneas anteriores los pacientes con diagnóstico de cáncer reportan niveles elevados de distrés emocional y estrés crónico que tiene implicancia en disturbios inmunológicos, particularmente en reducciones de células $\mathrm{T}$ y NK, un aumento elevado de citocinas pro-inflamatorias, tales como IL-6, IL-10 y IL-4, los cuales causan síntomas como depresión, alteraciones cognitivas, fatiga, dolor crónico e insomnio. Estas alteraciones inmunológicas vienen siendo demostradas en estudios científicos recientes (Kiecolt- 
Glaser et al., 2003; Witek-Janusek, Gabram \& Mathews, 2007).

Lengacher, Moscoso y colaboradores (2009) reportaron una significativa reducción en los síntomas de depresión y temor de recurrencia del tumor maligno en pacientes con cáncer de mama. Asimismo, estos investigadores demostraron que el grupo de pacientes asignados al grupo de tratamiento con la terapia de mindfulness presentó un aumento en los niveles de energía y funcionamiento físico en comparación con el grupo de control.
Los resultados reportados por nuestro equipo de investigación de la Universidad de South Florida acerca del programa TCCM en pacientes oncológicos son sumamente alentadores. En este sentido, el apoyo financiero que venimos recibiendo del Instituto Nacional de Salud de Estados Unidos para continuar nuestro programa de investigación en psiconeuroinmunología desarrollando estudios que examinen los aspectos emocionales, endocrinos e inmunológicos en pacientes con cáncer de mama, es testimonio de

\section{Figura 3}

\section{Efectos fisiológicos y psicológicos del mindfulness}

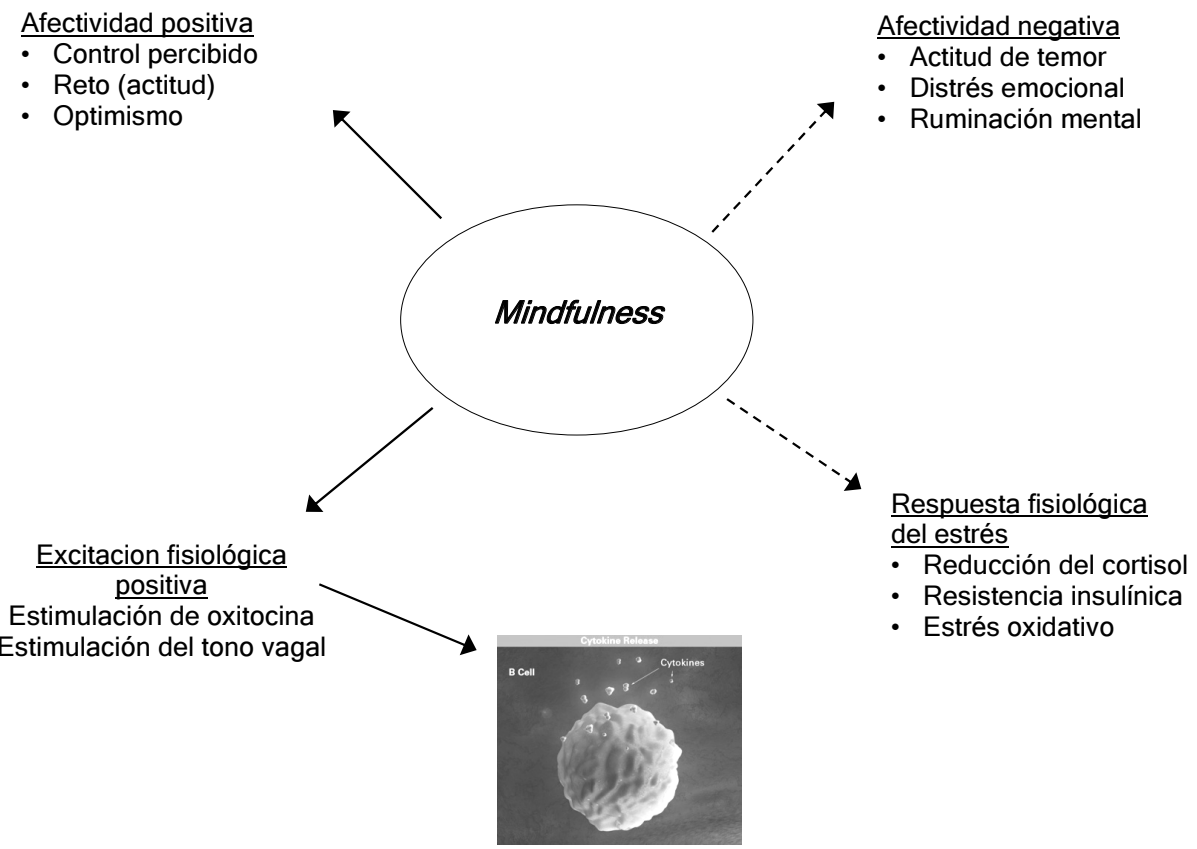

Citocinas antiinflamatorias 
un futuro promisorio para los programas terapéuticos, clínicos y educativos de reducción de estrés centrada en mindfulness.

\section{CONCLUSIONES}

El concepto del estrés ha evolucionado significativamente desde que Cannon y Selye iniciaran sus estudios en esta área de investigación. Actualmente, nuestra atención está centrada en el rol de los mediadores y moduladores del estrés crónico, y en lograr entender cómo estos factores pueden promover un adecuado proceso de adaptación por parte del individuo. Existe evidencia científica suficiente en relación con el impacto negativo del estrés crónico sobre el cerebro, los sistemas nervioso, endocrino e inmune y está claramente establecido que este impacto negativo representa un factor importante en la etiología y el progreso de un extenso número de enfermedades. Particular importancia para el mejor entendimiento del impacto del estrés crónico en la salud del individuo tienen los conceptos de estrés percibido y distrés emocional.

El lóbulo frontal del cerebro es el órgano central de percepción y respuesta a estresores, determinando las respuestas fisiológica, emocional y comportamental del estrés. La respuesta del organismo al estrés agudo es de naturaleza protectora y adaptativa, mientras que la respuesta al estrés crónico produ- ce un desbalance bioquímico, generando alteraciones físicas e inmunosupresoras que conducen al desarrollo de enfermedades inflamatorias, fatiga causada por el agotamiento de las glándulas suprarrenales, enfermedades metabólicas que incluyen obesidad, diabetes tipo II y enfermedades cardiovasculares. El cerebro y los sistemas nervioso, endocrino e inmune interactúan a través de rutas múltiples de tipo anatómico, hormonal y neuropéptico. Bajo condiciones normales, las interacciones entre estos sistemas conducen al mantenimiento del balance homeostático del organismo, y como resultado de ello una buena salud.

Una nueva visión acerca del estrés requiere tener en cuenta factores ambientales y estilos de vida del individuo. Es de particular importancia considerar un buen nivel de soporte social, un adecuado programa nutricional y de entrenamiento físico, la inclusión de programas de relajación y meditación, y poner énfasis en la eliminación del consumo de tabaco y reducción en el consumo de alcohol, calorías y grasas saturadas, particularmente cuando se experimentan estados de estrés crónico por un prolongado periodo de tiempo.

Presentamos un énfasis especial en el área de oncología describiendo el impacto emocional del cáncer y el rigor de sus tratamientos, los cuales afectan significativamente la calidad de vida del paciente y su familia, extendiendo estas dificultades por periodos muy largos, aun después de la culminación de 
los tratamientos correspondientes. Los pacientes que reciben el diagnóstico de cáncer reportan niveles elevados de distrés emocional y estrés crónico, lo cual es exacerbado por los rigurosos tratamientos, y ello tiene gran implicancia en los disturbios endocrinos e inmunológicos, particularmente en reducciones de células T, NK y un elevado aumento de citocinas pro-inflamatorias.

Finalmente, concluimos que el manejo del estrés crónico a través de la práctica de la TCCM permite el aprendizaje de la respuesta de relajación, así como un entendimiento más profundo de los síntomas fisiológicos, emocionales y comportamentales que caracterizan los estados de estrés crónico.

\section{REFERENCIAS}

Armeli, S.; Todd, M. \& Mohr, C. (2005). A daily process approach to individual differences in stress-related alcohol use. Journal of Personality, 73(6), 1-30.

Bandura, A. (1997). Self-efficacy: The exercise of control. Nueva York: W. H. Freeman.

Bishop, S. R. (2002). What do we really know about Mindfulnessbased stress reduction? Psychosomatic Medicine, 64, 71-83.

Cannon, W. B. (1915). Bodily changes in pain, hunger, fear, and rage. Boston: C. T. Branford Co., Inc.
Cannon, W. B. (1932). The wisdom of the body. Nueva York: W. W. Norton \& Co., Inc.

Carlson, L. E.; Speca, M.; Patel, K. D. $\&$ Goodey, E. (2003). Mindfulnessbased stress reduction in relation to quality of life, mood, symptoms of stress, and immune parameters in breast and prostate cancer outpatients. Psychosomatic Medicine, 65, 571-581.

Carlson, L. E.; Speca, M.; Patel, K. D. $\&$ Goodey, E. (2004). Mindfulnessbased stress reduction in relation to quality of life, mood, symptoms of stress and levels of cortisol, dehydroepiandrosterone sulfate (DHEAS) and melatonin in breast and prostate cancer outpatients. Psychoneuroendocrinology, 29, 448-474.

Carlson, L. E.; Speca, M.; Patel, K. D. $\&$ Faris, P. (2007). One year prepost intervention follow-up of psychological, immune, endocrine and blood pressure outcomes of Mindfulness-based stress reduction (MBSR) in breast and prostate cancer outpatients. Brain, Behavior and Immun., 21, 1038-1049.

Carlson, L. E.; Ursuliak, Z.; Goodey, E.; Angen, M., \& Speca, M. (2001). The effects of a Mindfulness meditation-based stress reduction program on mood and symptoms of stress in cancer outpatients: 6- 
month follow-up. Support Care Cancer, 9, 112-123.

Dallman, M. F. (2003). Chronic stress and obesity: A new view of 'comfort food'. Proc. Natl. Acad. Sci. USA, 100, 11696-11701.

Dhabhar, F. S. \& McEwen, B. S. (1997). Acute stress enhances while chronic stress suppresses cellmediated immunity in vivo: A potential role for leukocyte trafficking. Brain, Behavior, and Immunity, 11(4), 286-306.

Epel, E.; Lapidus, R. \& McEwen, B. (2000). Stress may add bite to appetite in women: A laboratory study of stress-induced cortisol and eating behavior. Psychoneuroendocrino logy, 26, 37-49.

Goldstein, D. S. \& Eisenhofer, G. (2000). Sympathetic nervous system physiology and pathophysiology in coping with the environment. En B.S. McEwen (Ed.). Coping with the environment: neural and endocrine mechanisms. Vol. 4, 21-43. Nueva York: Oxford University Press.

Kiecolt-Glaser, J. K.; McGuire, L. \& Robles, T. F. (2002). Psychoneuroimmunology: psychological influences on immune function and health. J. Consult. Clin. Psychol., 70, 537-547.

Kiecolt-Glaser, J. K.; Preacher, K. J.; MacCallum, R. C.; Atkinson, C.;
Malarkey, W. B. \& Glaser, R. (2003). Chronic stress and age-related increases in the proinflammatory cytokine IL-6. Proc. Natl. Acad. Sci. USA. 100, 9090-9095.

Koob, G. F. (1999). Corticotropin-releasing factor, norepinephrine, and stress. Biol. Psychiat., 46, 11671180.

Lazarus, R. S. (1993). Coping theory and research: Past, present, and future. Psychosomatic Medicine, 55(3), 234-247.

Lazarus, R. S. \& Folkman, S. (1984). Stress, appraisal, and coping. Nueva York: Springer.

Lengacher, C. A.; Johson-Mallard, V.; Post-White, J.; Moscoso, M. S.; Jacobsen, P. B.; Klein et al. (2009). Randomized controlled trial of Mindfulness-based stress reduction (MBSR) for survivors of breast cancer. Psycho-Oncology. DOI: 10.1002/pon. 1529.

MacPherson, A.; Dinkel, K. \& Sapolsky, R. (2005). Glucorticoids worsen excitotoxin-induced expression of pro-inflammatory cytokines in hippocampal cultures. Exper. Neurol., 194, 376-383.

Martin, J. L. \& Dean, L. (1993). Effects of AIDS-related bereavement and HIV-related illness on psychological distress among gay men - A 7-year longitudinal study 1985-1991. Journal of Consulting 
and Clinical Psychology, 661(1), 94-103.

McEwen, B. S. (1998). Protective and damaging effects of stress mediators. New England J. Med., 338, 171-179.

McEwen, B. S. (2001). Plasticity of the hippocampus: adaptation to chronic stress and allostatic load. Annals of the New York Academy of Sciences, 933, 265-277.

McEwen, B. S. (2006). Sleep deprivation as a neurobiologic and physiologic stressor, allostastis and allostatic load. Metabolism, 55, S20S23.

McEwen, B. S. (2007). The physiology and neurobiology of stress and adaptation, Central role of the brain. Physiol. Rev., 87, 873-904.

McEwen, B. S. \& Wingfield, J. C. (2003). The concept of allostasis in biology and biomedicine. Horm. \& Behav., 43, 2-15.

Moscoso, M. S. (1995). Manejo cognitivo-conductual del estrés en individuos VIH-1 seropositivos. Avances en Psicología Clínica Latinoamericana, 13, 39-53.

Moscoso, M. S. (2009). De la mente a la célula: Impacto del estrés en psiconeuroinmunoendocrinología. Revista Liberabit, 15 (2), 143-152.

Moscoso, M. S.; McCreary, D.; Goldenfarb, P.; Knapp, M. \& Rohr,
J. (1999). Construction of an inventory to measure emotional distress in cancer patients. PsychoOncology, 8(6), S4.

Moscoso, M. S.; McCreary, D.; Goldenfarb, P.; Knapp, M. \& Reheiser, E. (2000). A brief screening inventory to measure emotional distress in cancer patients. Psycho-Oncology, 9(5), S53.

Moscoso, M. S. \& Reheiser, E. C. (2010). Construct validity of the Emotional Distress Inventory in patients with cancer. Ansiedad $y$ Estres, 16 (1), 83-94.

Moscoso, M. S.; Reheiser, E. C. \& Hann, D. (2004). Effects of a brief Mindfulness-based stress reduction intervention on cancer patients. Psycho-Oncology, 13(1), S12.

Padgett, D. A. \& Glaser, R. (2003). How stress influences the immune response. Trends in Immunology, 24(8), 444-448.

Sapolsky, R. M. (1996). Why stress is bad for your brain. Science, 273, 749-750.

Sapolsky, R. M.; Romero, L. M. \& Munck, A. U. (2000). How do glucocorticoids influence stress responses? Integrating permissive, suppressive, stimulatory, and preparative actions. Endocrine Review, 21, 55-89. 
Selye, H. (1936). A syndrome produced by diverse nocuous agents. Nature, 138, 32 .

Speca, M.; Carlson, L. E.; Goodey, E. \& Angen, M. (2000). A randomized, wait-list controlled clinical trial: the effect of a Mindfulness meditationbased stress reduction program on mood and symptoms of stress in cancer outpatients. Psychosomatic Medicine, 62, 613-622.

Spielberger, C. D. \& Moscoso, M. S. (1996). Reacciones emocionales del estrés: Ansiedad y cólera. Avances en Psicología Clínica Latinoamericana, 14, 59-81.

Steinman, L. (2004). Elaborate interactions between the immune and nervous systems. Nat. Immunol, 5, 575-581.

Sterling, P. \& Eyer, J. (1988). Allostasis, a new paradigm to explain arousal pathology. En S. Fisher \& J. Reason (Eds.). Handbook of life stress, cognition and health (pp. 629-649). Nueva York: John Wiley \& Sons.

Taub, D. (2008). Novel Connections between the Neuroendocrine and Immune Systems: The Ghrelin Immunoregulatory Network. Vitam. Hor., 77, 325-346.

Taylor, S. E.; Klein, L. C.; Lewis, B. P.; Gruenewald, T. L.; Gurung, R. A. R. \& Undergraff, J. A. (2000). Biobehavioral responses to stress in females: tend-and-befriend, not fight-or-flight. Psychological Review, 107, 4121-4129.

Venter, C. (2008). Genes have very little impact on life outcomes. India Today, 3.

Witek-Janusek, L.; Gabram, S. \& Mathews, H. L. (2007). Psychologic stress, reduced NK cell activity, and cytokine dysregulation in women experiencing diagnostic breast biopsy. Psychoneuroendocrinology, 32, 22-35. 